\title{
Treatment of advanced melanoma - A changing landscape
}

\author{
Adriana Hepner ${ }^{1}$, Alessandra Salgues ${ }^{1}$, Carlos A. dos Anjos $^{1}$, Marina Sahade $^{1,2}$, Veridiana P. Camargo ${ }^{1,2}$, \\ Bernardo Garicochea ${ }^{1}$, Alexander N. Shoushtari ${ }^{3,4}$, Michael A. Postow ${ }^{3,4}$, Gustavo S. Fernandes ${ }^{1}$, Rodrigo R. Munhoz ${ }^{1,2 *}$ \\ ${ }^{1}$ Oncology Center, Hospital Sírio-Libanês, São Paulo, SP, Brazil \\ ${ }^{2}$ Medical Oncology Service, Instituto do Câncer do Estado de São Paulo, Universidade de São Paulo, São Paulo, SP, Brazil \\ ${ }^{3}$ Melanoma and Immunotherapeutics Service, Memorial Sloan Kettering Cancer Center, New York, NY, USA \\ ${ }^{4}$ Weill Cornell Medical College, New York, NY, USA
}

Article received: $12 / 7 / 2016$ Accepted for publication: 2/5/2017

*Correspondence: Address: Rua Dona Adma Jafet, 91 bloco $\mathrm{A}, 2^{\circ}$ andar São Paulo, SP - Brazil Postal code: $01308-050$ munhozrs@gmail.com

http://dx.doi.org/10.1590/1806-9282.63.09.814

\section{SUMMARY}

Following decades of relative ostracism, advances in the treatment of melanoma have brought a new reality for patients, physicians and researchers. While antibodies targeting molecules involved in the modulation of the interaction between melanoma and immune cells changed the meaning of the term "cancer immunotherapy," a better characterization of the molecular aberrations involved in melanoma carcinogenesis prompted the development of inhibitors of the mitogen-activated protein kinase pathway (MAPK) that also led to significant improvements both in response rates and survival. As a result, new drugs have been approved for clinical use in the United States and Europe, including the immune-checkpoint blockers ipilmumab, pembrolizumab and nivolumab, the oncolytic herpesvirus talimogene laherparepvec, and the targeted-agents vemurafenib, dabrafenib, cobimetinib and trametinib. In this article, we review the results of studies that brought new approaches to the bedside and discuss how these developments are being incorporated into the care of patients in Brazil.

Keywords: melanoma, anti-PD1, anti-CTLA4, BRAF, MEK.

\section{INTRODUCTION}

Although it represents only $1 \%$ of all cutaneous malignancies, melanoma is still a challenge to public health due to its high metastatic potential and mortality. ${ }^{1}$ Over the past decades, the number of cases of melanoma has increased dramatically, faster than any other type of cancer. ${ }^{2}$ In the United States, 76,380 new cases and more than 10,000 deaths related to melanoma are expected in 2016, accounting for the vast majority of skin cancer deaths. ${ }^{1}$ In Latin America, data regarding the incidence and prevalence of this disease are scarce, ${ }^{3}$ and 5,670 new cases are estimated in Brazil in 2016 according to the Instituto Nacional do Câncer (Inca). ${ }^{4}$

While patients with early diagnosis have 5 -year survival rates around $90 \%$, historically, this number decreases to $10 \%$ in patients with advanced melanoma, with a median survival of 6 to 12 months. ${ }^{1,2}$

Even though surgery and radiotherapy may have a role in the management of metastatic disease in selected situations, systemic therapy is the mainstay of treatment for most patients. ${ }^{5}$ For over three decades, dacarbazine was the most commonly used cytotoxic agent, resulting in objective responses in approximately $10 \%$ of the cases and with an arguable impact on overall survival, with approximately $20-25 \%$ of the patients alive at 12 months. ${ }^{2,5}$ Other agents, such as vinblastine, cisplatin/carboplatin and taxanes, either used in combinations or in monotherapy, showed only short-lived benefits. ${ }^{5}$ Non-selective forms of immunotherapy, including high dose interleukin-2 (IL-2) or biochemotherapy, had their widespread use hampered by significant toxicity and objective (albeit durable and potentially curative) responses limited to a small proportion of individuals, despite serving as a proof-of-concept that melanoma cells could be controlled or eradicated by the immune system. ${ }^{6}$

In the past decades, however, progress has been made in the understanding of both melanoma pathogenesis and the interaction between cancer and immune cells. The demonstration of aberrant activation of the mitogenactivated protein kinase pathway (MAPK) paved the way for the development of so-called targeted therapies, ${ }^{7}$ including the currently available V-Raf Murine Sarcoma 
Viral Oncogene Homolog B (BRAF) and mitogen-activated protein kinases enzyme (MEK) inhibitors (Figure 1). ${ }^{8,9}$ In parallel, the manipulation of the immune system by blocking ligands and receptors that act as regulators of the $\mathrm{T}$ cell activation, the so-called immune checkpoints, exemplified by the cytotoxic $\mathrm{T}$ lymphocyte associated antigen 4 (CTLA-4) and programmed cell death protein 1 (PD1) and its ligand (PD-L1) have become important strategies to control advanced tumors (Figure 2). As a result, objective responses can be seen in more than 50\% of the cases and the $25-35 \%$ probability of a patient being alive has transitioned from 12 months in the era of conventional chemotherapy to $48-60$ months. ${ }^{10}$

While survival of patients with advanced melanoma has been considerably improved over a relatively short interval (Figure 3), the near future probably holds even more consistent advances. In this article, we will review the results of studies that led to a change in the management of patients with advanced melanoma and discuss how these new agents are being incorporated into treatment algorithms.

\section{Manipulating signaling pathways in MELANOMA - THE USE OF BRAF AND MEK INHIBITORS}

Activating mutations of the BRAF gene, which is an upstream component of the growth-promoting MAPK pathway (Figure 1), are found in approximately 40 to $60 \%$ of patients with metastatic melanoma. ${ }^{79}$ In about 75 to $80 \%$ of the cases, the mutation occurs in the region that encodes the kinase domain and consists of the substitution of glutamic acid for valine at amino acid 600 (the V600E muta-

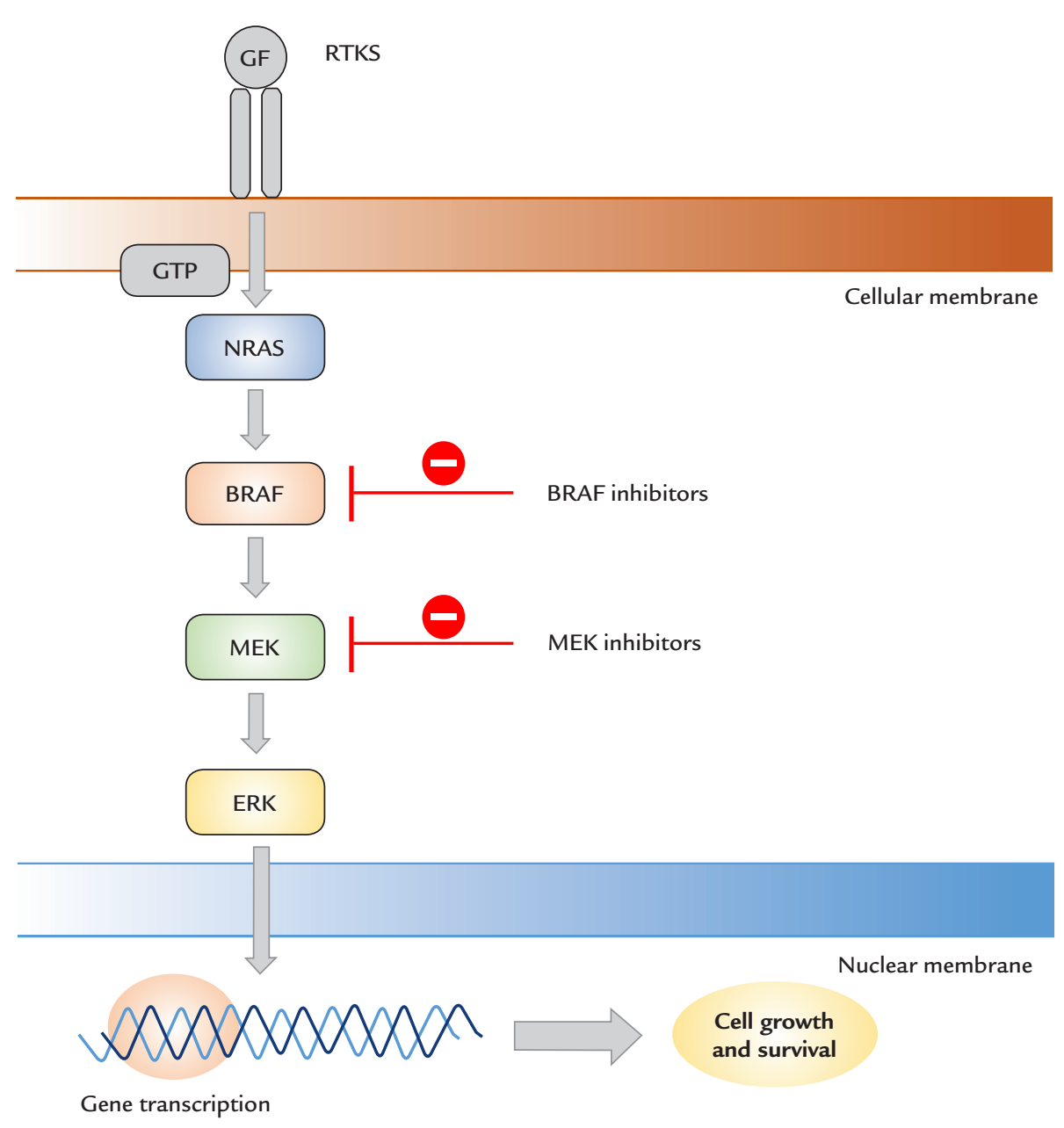

FIGURE 1 The MAPK pathway and the role of BRAF and MEK inhibitors.

RTKS: receptor tyrosine kinases; GF: growth factor; GTP: guanosine triphosphate; NRAS: neuroblastoma RAS viral oncogene homolog; BRAF: V-Raf murine sarcoma viral oncogene homolog B; MEK: mitogen-activated protein kinases enzyme; ERK: extracellular signal-regulated kinase. 


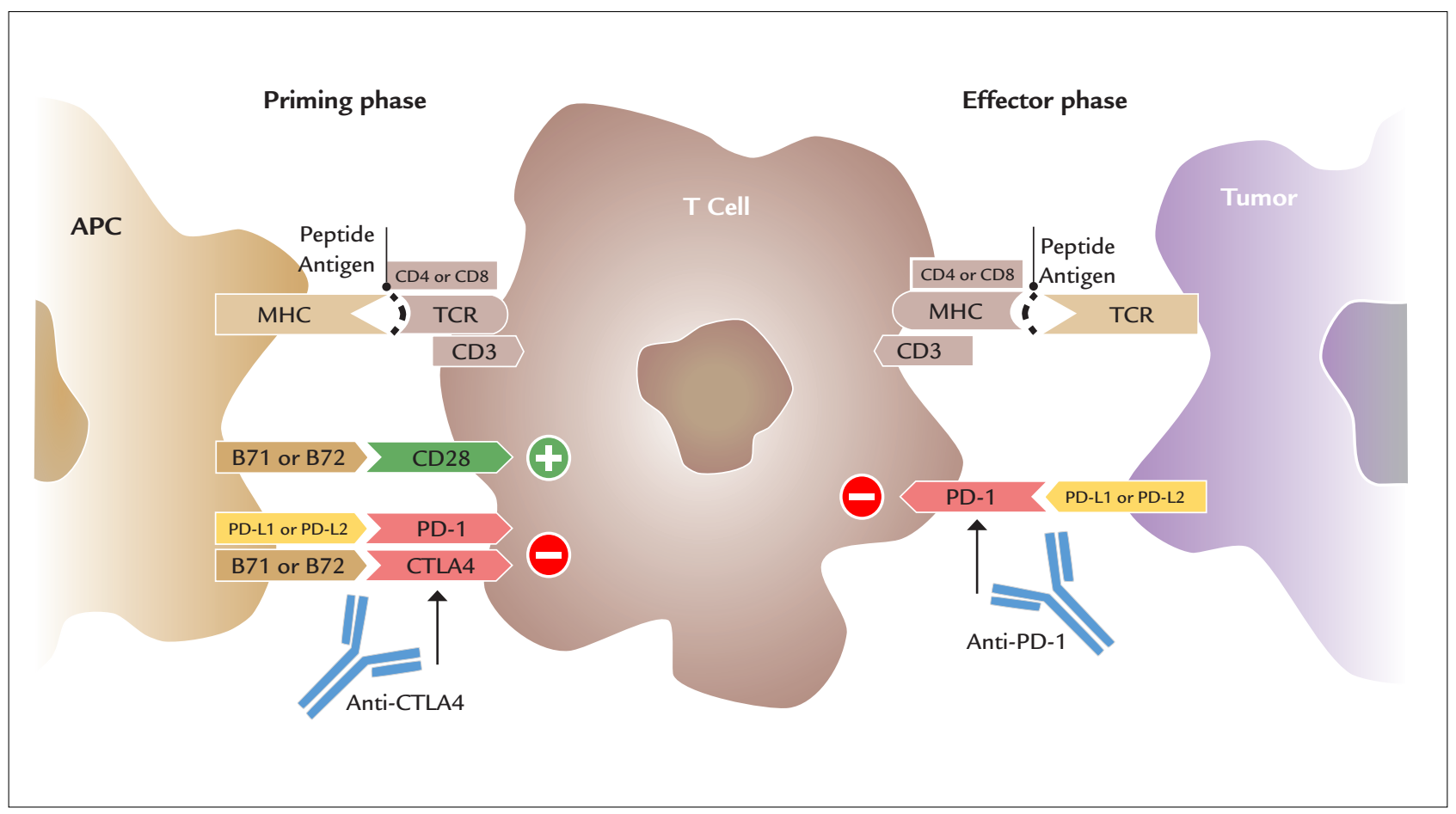

FIGURE 2 CTLA-4 and PD-1/PD-L1 in the immune synapse.

MHC: major histocompatibility complex; TCR: T cell receptor; CD: cluster of differentiation; B7.1 and B7.2 proteins; CTLA4: cytotoxic T lymphocyte associated antigen 4; PD1: programmed cell death protein 1; PD-L1: programmed death-ligand 1; PD-L2: programmed death-ligand 2.

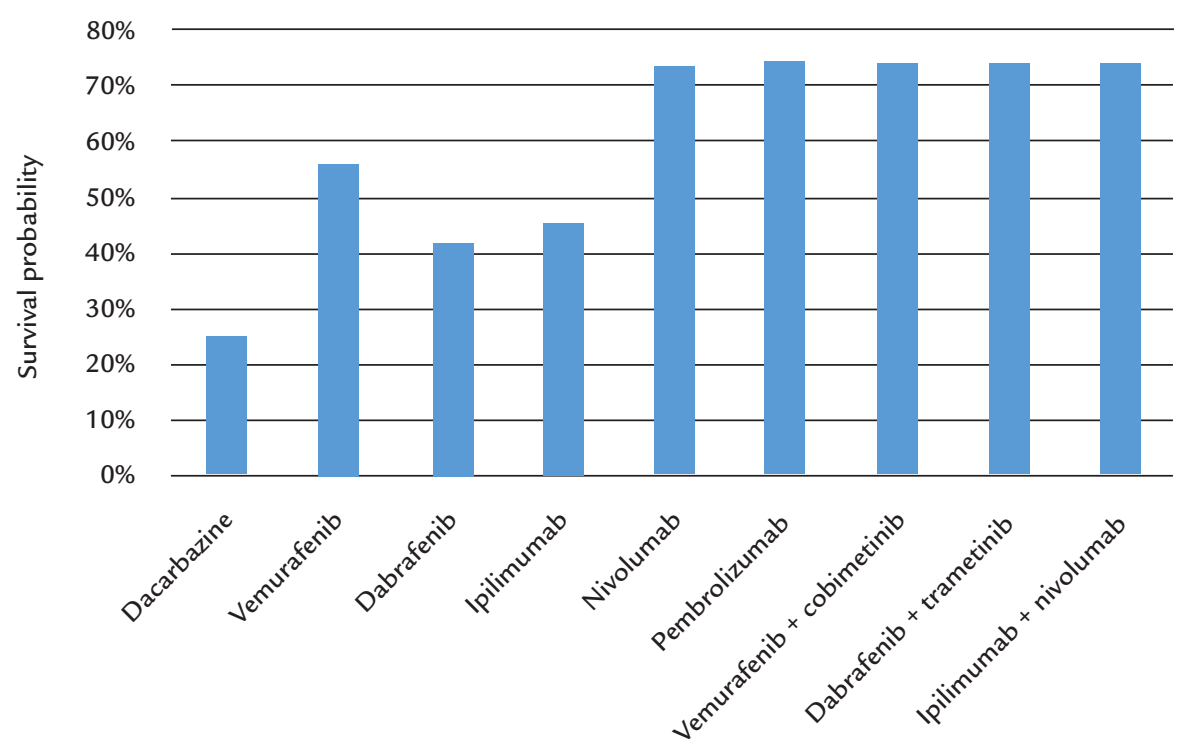

Treatment

FIGURE 3 12-month overall survival rates across different studies. 
tion). ${ }^{11}$ In another $20 \%$ of the tumors harboring a BRAF mutation, an alternate substitution of lysine for valine occurs (the V600K mutation). ${ }^{9,11}$ Therapeutic manipulation of the aberrantly activated MAPK pathway as a result of those specific mutations has been proven to be an important approach for the treatment of advanced melanoma patients.

The first investigated anti-BRAF agent was the multikinase inhibitor sorafenib, which failed to show meaningful clinical activity as a single agent or in combination with chemotherapy. ${ }^{12}$ Since then, vemurafenib and dabrafenib, more potent and selective BRAF inhibitors (BRAFi), were developed. The clinical efficacy of orallyadministered BRAF inhibitor vemurafenib in patients with BRAF V600-mutated melanoma has been established in the phase 3 BRIM-3 trial, in which 675 patients with metastatic/unresectable disease were randomly assigned to either vemurafenib (960 $\mathrm{mg}$ twice daily) or dacarbazine $\left(1,000 \mathrm{mg} / \mathrm{m}^{2}\right.$ given intravenously every three weeks). The updated objective response rate (ORR) confirmed by an independent review was $47 \%$ among patients treated with vemurafenib compared to $9 \%$ in the dacarbazine arm. ${ }^{13}$ After a median follow-up of 12.5 months, vemurafenib resulted in a statistically significant improvement in overall survival (OS) (13.6 vs. 9.7 months; HR 0.70, 95CI 0.57-0.87; $\mathrm{p}=0.0008$ ), with $56 \%$ of the patients alive at 12 months. Progression-free survival (PFS) was also significantly prolonged (6.9 vs. 1.6 months; HR 0.38, 95CI 0.32-0.46; $\mathrm{p}<0.0001$ ). ${ }^{13}$

Dabrafenib also demonstrated significant activity in advanced melanoma and has been approved for clinical use. In a phase 3 trial, dabrafenib (150 mg taken orally twice daily) was compared to dacarbazine $\left(1,000 \mathrm{mg} / \mathrm{m}^{2}\right.$ given intravenously every three weeks) in 250 patients with unresectable stage III or stage IV melanoma harboring a BRAF V600E mutation. Dabrafenib significantly prolonged the median PFS (which was the primary endpoint of the study) (5.1 vs. 2.7 months; HR 0.33; 95CI 0.20-0.54; $\mathrm{p}<0.0001$ ), resulting in an ORR of $50 \%$ versus $6 \% .{ }^{14}$ OS was updated following a median follow-up of 13 to 15 months; while the difference in survival was not statistically significant, crossover was permitted between the two groups and occurred in $57 \%$ of the patients initially treated with dacarbazine. ${ }^{15}$

The most frequent toxicities associated with BRAF inhibition were dermatologic (rash, photosensitivity and hyperkeratosis), arthralgia, fatigue, nausea and diarrhea, although differences in the toxicity profile of dabrafenib and vemurafenib occur. Cutaneous squamous cell carcinomas (SCC) or keratoacanthoma may develop in up to $25 \%$ of patients treated with vemurafenib. ${ }^{13}$ Conversely, febrile reactions/pyrexia and severe hyperglycemia are more frequent with dabrafenib and require attention. ${ }^{14,15}$
Nevertheless, despite initial response, secondary resistance often limits the benefit of single-agent BRAF inhibitors, and underlying mechanisms involve reactivation of the MAPK pathway in almost $70 \%$ of the cases. ${ }^{16}$ Hence, blockade of an immediate downstream signaling component in the MAPK pathway, MEK, could potentially result in significant antitumor effect. Initially tested as single agent for patients who had not received prior treatment with a BRAFi, trametinib, a selective blocker of MEK1 and MEK2, was approved based on a survival advantage in the phase 3 METRIC study (6-month survival rate $81 \%$ vs. $67 \%$; 95 CI $0.32-0.92 ; \mathrm{p}=0.01$ ). Objective responses, despite comparing favorably to dacarbazine (ORR $8 \%$ ), occurred in only $22 \%$ of the patients. ${ }^{17}$ The rationale for the development of trials addressing dual MAPK pathway blockade was based on the possibility of minimizing the toxicity associated with paradoxical activation of the MAPK pathway in the setting of BRAF inhibition, delaying treatment resistance and enhancing the antitumor effect. Based on early evidence that simultaneous, rather than sequential administration, could represent the optimal approach, subsequent phase 3 studies evaluated BRAFi (vemurafenib or dabrafenib) given concurrently with MEKi (cobimetinib or trametinib). ${ }^{18,19}$

The combination dabrafenib (150 mg twice daily) plus trametinib ( $2 \mathrm{mg}$ once daily) was compared to singleagent dabrafenib or vemurafenib in the COMBI-d and COMBI-v trials, respectively. ${ }^{18,20,21}$ Both studies have consistently shown increased response rates, and gains in PFS and OS favoring the use of the combination (Table 1 ), with updated 3 -year survival rates of 44 and $45 \%{ }^{22,23}$ In addition, the two trials confirmed that the incidences of cutaneous squamous cell carcinoma and keratoacanthoma were significantly decreased with the combination treatment by almost one third. ${ }^{18,20,21}$ In the coBRIM trial, another BRAFi/MEKi association was studied in 495 patients with previously untreated, BRAF-mutated, advanced melanoma. In this phase 3 trial, patients were randomly assigned to vemurafenib (960 mg given twice daily continuously, on days 1 to 28 ) plus cobimetinib (60 mg daily on days 1 to 21 , followed by a 7 -day interval) in 28-day cycles, or to vemurafenib plus placebo. Median PFS, the primary endpoint of the study, was significantly prolonged in the combination group (median PFS 12.3 vs. 7.2 months, HR 0.58; 95CI 0.46-0.72; $\mathrm{p}<0.0001$ ). More importantly, combined blockade resulted in gains in overall survival (22.3 vs. 17.4 months, HR 0.70; 95CI $0.55-0.90 ; \mathrm{p}=0.005)$ and ORR (70\% vs. $50 \%) .{ }^{19}$ These results have led to regulatory approvals of the aforementioned combinations. 
TABLE 1 Selected published clinical trials.*

\begin{tabular}{|c|c|c|c|c|c|c|c|}
\hline Study & $\begin{array}{l}\text { Author, } \\
\text { year }\end{array}$ & Phase & $\mathbf{N}$ & Intervention & ORR (\%) & mPFS (months) & Survival data \\
\hline \multicolumn{8}{|c|}{ Immune-checkpoint blockade } \\
\hline- & Hodi, 2010 & 3 & 676 & $\begin{array}{l}\text { Ipilimumab } 3 \text { mg/kg vs. } \\
\text { Ipilimumab } 3 \text { mg/kg+gp } \\
100 \text { vs. gp100 }\end{array}$ & $\begin{array}{l}10.9 \times 5.7 \\
\times 1.5\end{array}$ & $2.86 \times 2.76 \times 2.76$ & $\begin{array}{l}\mathrm{mOS} 10.1 \mathrm{mo} \times 10.0 \mathrm{mo} \\
\times 6.4 \mathrm{mo}\end{array}$ \\
\hline - & Robert, 2011 & 3 & 502 & $\begin{array}{l}\text { Ipilimumab } 10 \text { mg/kg+DTIC } \\
\text { vs. DTIC }\end{array}$ & $15.2 \times 10.6$ & Not available & $5 y$ OS $18.2 \%$ x $8.8 \%$ \\
\hline KEYNOTE 001 & Ribas, 2016 & 1 & 655 & Pembrolizumab & 33 & 5,2 & mOS $23 \mathrm{mo}$ \\
\hline KEYNOTE 002 & Ribas, 2015 & 3 & 540 & $\begin{array}{l}\text { Pembrolizumab } 2 \text { mg/kg vs. } \\
\text { Pembrolizumab } 10 \text { mg/kg vs. } \\
\text { CT }\end{array}$ & $38 \times 46 \times 8$ & $4.2 \times 5.6 \times 2.6$ & NR \\
\hline KEYNOTE 006 & Robert, 2015 & 3 & 834 & $\begin{array}{l}\text { Pembrolizumab q } 14 d x \\
\text { Pembrolizumab q } 21 d x \\
\text { Ipilimumab }\end{array}$ & $\begin{array}{l}33.7 \times \\
32.9 \times \\
11.9\end{array}$ & $5.5 \times 4.1 \times 2.8$ & $2 y$ OS $55 \%$ x $55 \% \times 43 \%$ \\
\hline- & Topalian, 2014 & 1 & 107 & Nivolumab & 31 & 3,7 & mOS $16.8 \mathrm{mo}$ \\
\hline CheckMate 037 & Weber, 2015 & 3 & 405 & Nivolumab vs. CT & $32 \times 5$ & $4.7 \times 4.2$ & NR \\
\hline CheckMate 066 & Robert, 2015 & 3 & 418 & Nivolumab vs. Dacarbazine & $40 \times 13.9$ & $5.1 \times 2.2$ & $\mathrm{NR} \times 10.8 \mathrm{mo}$ \\
\hline CheckMate 069 & Postow, 2015 & 2 & 142 & $\begin{array}{l}\text { Ipilimumab/Nivolumab vs. } \\
\text { Ipilimumab }\end{array}$ & $61 \times 11$ & $N R \times 4.4$ & NR \\
\hline CheckMate 067 & Larkin, 2015 & 3 & 945 & $\begin{array}{l}\text { Ipilimumab/Nivolumab vs. } \\
\text { Nivolumab vs. Ipilimumab }\end{array}$ & $\begin{array}{l}57.6 \times 43.7 \\
\times 19\end{array}$ & $11.5 \times 6.9 \times 2.9$ & NR \\
\hline MAPK pathway & blockade & & & & & & \\
\hline BRIM 3 & $\begin{array}{l}\text { McArthur, } \\
2014\end{array}$ & 3 & 675 & Vemurafenib vs. Dacarbazine & $48 \times 5$ & $6.9 \times 1.6$ & $\mathrm{mOS} 13.6 \mathrm{mo} \times 9.7 \mathrm{mo}$ \\
\hline BREAK 3 & $\begin{array}{l}\text { Hauschild, } \\
2012\end{array}$ & 3 & 250 & Dabrafenib vs. Dacarbazine & $50 \times 6$ & $5.1 \times 2.7$ & NR \\
\hline METRIC & Flaherty, 2012 & 3 & 322 & Trametinib vs. CT & $22 \times 8$ & $4.8 \times 1.5$ & 6 mo OS $81 \% \times 67 \%$ \\
\hline COBRIM & Ascierto, 2016 & 3 & 495 & $\begin{array}{l}\text { Vemurafenib + Cobimetinib } \\
\text { vs. Vemurafenib }\end{array}$ & $70 \times 50$ & $12.3 \times 7.2$ & $\operatorname{mOS} 22.3 \mathrm{mo} \times 17.4 \mathrm{mo}$ \\
\hline COMBI-v & Robert, 2015 & 3 & 704 & $\begin{array}{l}\text { Dabrafenib + Trametinib vs. } \\
\text { Vemurafenib }\end{array}$ & $64 \times 51$ & $11.4 \times 7.3$ & $\operatorname{mOS} 25.6 \mathrm{mo} \times 18.3 \mathrm{mo}$ \\
\hline COMBI-d & Long, 2015 & 3 & 423 & $\begin{array}{l}\text { Dabrafenib + Trametinib vs. } \\
\text { Dabrafenib }\end{array}$ & $69 \times 53$ & $11.0 \times 8.8$ & $\mathrm{mOS} 25.1 \mathrm{mo} \times 18.7 \mathrm{mo}$ \\
\hline
\end{tabular}

*Data extracted from published manuscripts.

mo: months; N: number of patients enrolled; ORR: objective response rate; mPFS: median progression-free survival; OS: overall survival data; CT: chemotherapy; DTIC: dacarbazine; NR: not reached.

Besides alterations involving BRAF, other melanoma gene mutations have been identified, which can also offer significant therapeutic insights. NRAS, an upstream effector of the MAP and PI3K pathways, is mutated in about $20 \%$ of the cases. ${ }^{9,24}$ Other less common mutations occur in NF1 and c-KIT. ${ }^{7,9}$ Initial results of a phase 3 trial comparing binimetinib, a MEKi, to dacarbazine in patients with advanced NRAS mutation tumors, showed an increase in PFS (median PFS 2.8 vs. 1.5 months; HR 0.62; 95CI $0.47-0.80 ; \mathrm{p}<0.001)$. In this trial, there was no significant difference in overall survival (11 vs. 10 months), although survival data were still immature. ${ }^{24}$ Although infrequent, c-KIT mutations can be found in acral and mucosal melanomas; in several case reports, a rapid, but transient response was achieved with imatinib mesylate, a small molecule inhibitor of KIT and other tyrosine kinases. ${ }^{25,26}$ These observations were confirmed in subsequent prospective, non-comparative phase 2 studies, in which imatinib resulted in response rates of approximately $20 \%$, despite relatively short PFS intervals ranging from 2.8 to 3.7 months. ${ }^{27,28}$ Taken together, although the benefit of targeted approaches in patients with melanoma 
harboring non-BRAF mutations has been limited, these results serve as a proof of concept for future molecularlydriven treatment strategies.

\section{USING THE IMMUNE SYSTEM TO FIGHT MELANOMA - THE GROWING FIELD OF IMMUNOTHERAPY}

Using the immune system to fight cancer has evolved from a "future perspective" to one of the most practicechanging breakthroughs in oncology in recent years, yielding the possibility of long-term clinical benefit and prolonged survival. Indeed, the development of monoclonal antibodies targeting co-receptors involved in escape mechanisms has shown exceptional results for the treatment of advanced melanoma.

The immune system can be divided into innate and adaptive immunity. The innate immune system is the initial defense against foreign antigens, which includes dendritic cells (DC), macrophages, neutrophils, basophils, eosinophils, natural killer cells (NK) and mast cells. These cells, once activated, release stimulatory cytokines that recruit additional elements of the immune response. The adaptive immune system is an antigen-specific response, dependent mainly on the antigen presenting cells (APCs), which process antigens and present them via mixed histocompatibility complex (MHC) class I and II molecules, to $\mathrm{T}$ cells through the T-cell receptor (TCR). Activated T cells, then differentiate into distinct functional phenotypes (exemplified per Th1, Th2 and Tregs), release cytokines and recruit effector cells, producing the T-cellmediated response. ${ }^{29}$

Recently, ligands and co-receptors expressed on $\mathrm{T}$ cells, APC and tumor cells have been identified as potential targets for immunotherapy, due to their critical role as immune suppressors at the tumor microenvironment. These ligands and receptors, because of their function as regulators of the $T$ cell activation and tolerance, have been termed "immune checkpoints" (Figure 2). Monoclonal antibodies directed against CTLA-4 and PD-1 and its ligand (PD-L1) illustrate successful approaches for the treatment of advanced melanoma. ${ }^{30}$

In the priming phase or early phase of immune activation, the CTLA-4 receptor is induced on T lymphocytes as negative regulator of $\mathrm{T}$ cell response, competing with $\mathrm{CD} 28$ for binding to B7 molecule on the antigen presenting cells. ${ }^{30}$ Ipilimumab, a monoclonal antibody that targets CTL4, was the first checkpoint inhibitor approved for clinical use in metastatic melanoma patients in 2011, based on survival gains in both first and second-line settings and response rates of $10-15 \%{ }^{31}$ In the largest combined analysis of 1,861 patients treated with ipilimumab in phase 2 and
3 trials, a plateau seen in the survival curve confirmed the possibility of sustained benefits, with $21 \%$ of those patients surviving beyond three years. ${ }^{32}$ Of note, a recently-presented randomized trial comparing ipilimumab at $3 \mathrm{mg} / \mathrm{kg}$ versus $10 \mathrm{mg} / \mathrm{kg}$ demonstrated an improvement in overall survival in the group of patients treated with higher doses (mOS 15.7 m vs. 11.5 m; HR 0.84; 95CI 0.70-0.99; $\mathrm{p}=0.04$ ), accompanied by an increase in the rates of grade 3-5 treatment-related toxicities ( $34.3 \%$ vs. $18.5 \%$ ). ${ }^{33}$

Despite this initial success with CTLA-4 blockade, even more compelling results were seen with anti-PD1 agents pembrolizumab and nivolumab. The PD-1 receptor is expressed by activated $\mathrm{T}$ cells and binds to its ligands PD-L1 and PD-L2, resulting in abrogation of T cell-mediated responses and preventing the recognition and killing by cytotoxic cells. ${ }^{30}$

In phase 1 trials, ORR around $30 \%$ and median OS of 23 months and 16.8 months were seen with pembrolizumab and nivolumab, respectively. ${ }^{34,35}$ These agents were initially approved for patients following progression on ipilimumab (and a BRAF inhibitor, in patients with melanoma harboring a BRAF mutation) based on the results of trials that demonstrated both favorable efficacy in comparison to cytotoxic chemotherapy. In the KEYNOTE 002 randomized phase 2 trial, 540 patients with ipilimumab-refractory disease were randomly assigned to pembrolizumab ( $2 \mathrm{mg} / \mathrm{kg}$ every three weeks), pembrolizumab (10 mg/kg every three weeks) or chemotherapy. The six-month progression-free rate, the primary endpoint of the study, was 34,38 , and $16 \%$, respectively (pembrolizumab $2 \mathrm{mg} / \mathrm{kg}$ versus chemotherapy; HR 0.57; 95CI 0.45-0.73; $\mathrm{p}<0.0001$; and pembrolizumab $10 \mathrm{mg} / \mathrm{kg}$ versus chemotherapy HR $0.50,95 \mathrm{CI} 0.39-0.64$; $\mathrm{p}<0.0001$ ), with objective responses occurring in $21-26 \%$ of the cases. ${ }^{36}$ Similarly, in the phase 3 CheckMate 037 study, 405 previously treated patients received either nivolumab or investigator's choice of chemotherapy. Objective responses were reported in $31.7 \%$ in the nivolumab group vs. $10.6 \%$ in the chemotherapy group, and less toxic effects were seen with nivolumab (rate of grade 3-4 adverse events: $9 \%$ vs. $32 \%) .{ }^{37}$

In the first-line setting, both pembrolizumab and nivolumab showed superiority in terms of efficacy and tolerability when compared to ipilimumab, and were rapidly incorporated into clinical practice for treatment-naïve patients. In the phase III KEYNOTE-006 trial, $834 \mathrm{pa}-$ tients received either pembrolizumab $10 \mathrm{mg} / \mathrm{kg}$ every two weeks, pembrolizumab $10 \mathrm{mg} / \mathrm{kg}$ every three weeks until disease progression or ipilimumab $3 \mathrm{mg} / \mathrm{kg}$ every three weeks for four doses. The trial demonstrated sig- 
nificant improvement in 2-year survival rates for both pembrolizumab regimens versus ipilimumab $155 \%$ in both pembrolizumab arms vs. 43\%; HR 0.68; 95CI 0.53$0.87 ; \mathrm{p}=0.0008$ and HR 0.68; 95CI 0.53-0.87; $\mathrm{p}=0.0008$ for 2 week and 3 week schedules compared to ipilimumab, respectively). The 6-month PFS rate, a co-primary endpoint, was $47.3 ; 46.4$ and $26.5 \%$ respectively, with a $\mathrm{HR}$ for disease progression for pembrolizumab versus ipilimumab of 0.58 ; $\mathrm{p}<0.001$ for both 2 and 3 -week regimens. ORR were 37, 36 and $13 \%$ for the same treatment arms. ${ }^{38,39}$

The longest follow-up data of melanoma patients on treatment with anti-PD1 therapy comes from the phase $1 / 2$ dose escalation expansion cohort of nivolumab in 107 heavily pretreated advanced melanoma patients. The median overall survival was 17 months, but treated patients achieved an impressive 5 -year survival rate of $34 \% .^{35}$

The combined administration of an anti-CTLA- 4 and anti-PD1 antibodies was tested in a randomized phase 2 trial that accrued 142 treatment-naive patients, with approximately $75 \%$ of BRAF "wild-type" (wt) tumors. Despite the increased incidence of grade 3 or 4 adverse events (54\% vs. $24 \%$ ), the combination of nivolumab and ipilimumab resulted in higher ORR in the BRAFwt population when compared to ipilimumab monotherapy, which was the primary endpoint (ORR in BRAFwt patients: 60\% vs. $11 \%){ }^{40,41}$ The hypothesis that combined immune-checkpoint blockade could result in improved outcomes was further tested in the CheckMate 067 phase 3 trial. In this study, 945 patients were randomized to receive nivolumab $1 \mathrm{mg} / \mathrm{kg}$ plus ipilimumab $3 \mathrm{mg} / \mathrm{kg}$ for four doses followed by nivolumab, nivolumab $3 \mathrm{mg} / \mathrm{kg}$ plus placebo or ipilimumab $3 \mathrm{mg} / \mathrm{kg}$ plus placebo. PFS and overall survival were the co-primary endpoints of the trial and the results were updated after a median follow-up of 18 months. The ORR was $19 \%$ for ipilimumab monotherapy compared with $57.6 \%$ for nivolumab plus ipilimumab $(\mathrm{p}<0.001)$ and $43.7 \%$ for nivolumab alone $(\mathrm{p}<0.001)$. The combination therapy was associated with a $58 \%$ relative reduction in the risk of disease progression when compared to ipilimumab alone (HR 0.42; 99.5CI 0.31-0.57; $\mathrm{p}<0.001$ ); similarly, nivolumab monotherapy resulted in a relative risk reduction of $45 \%$ also compared with ipilimumab alone (HR 0.55; 99.5CI 0.43-0.76; $\mathrm{p}<0.001$ ). ${ }^{41}$ Although the study was not powered for the direct comparison of nivolumab plus ipilimumab vs. nivolumab, an exploratory analysis showed that combination therapy reduced the risk for progression by $24 \%$ compared with nivolumab monotherapy (HR 0.76; 95CI 0.60-0.92). ${ }^{41,42}$ Grade 3 and 4 adverse events for the combination, nivolumab alone, and ipilimumab alone were 55,16 , and $27 \%$, respectively and treatment discontinuation due to treatment toxicities were more frequent in the combination arm. ${ }^{42}$ Longer follow-up and mature overall survival data are expected. Due to the high incidence of immune-mediated adverse events demonstrated in the setting of combined blockade, alternative treatment regimens are being investigated in an attempt to enhance the tolerability. As an example, the phase 1 KEYNOTE-029 trial combined "low dose" ipilimumab ( $1 \mathrm{mg} / \mathrm{kg}$ given every 3 weeks for 4 doses) with a standard dose of pembrolizumab $(2 \mathrm{mg} / \mathrm{kg}$ given every 3 weeks); ORR in this study was $57 \%$, and grade $3-4$ toxicities occurred in $42 \%$ of the cases. ${ }^{43}$

While PD-1 blockers, either in monotherapy or in combination with ipilimumab, became the standard of care for patients with BRAFwt tumors, the best treatment to be given upfront remains to be determined for those with tumors harboring a BRAF mutation, and results of ongoing studies looking at the best sequence and combinations of BRAF/MEK inhibition and immune-checkpoint blockade are eagerly awaited, as discussed below. ${ }^{44}$

\section{FUTURE PERSPECTIVES}

The increasing understanding of the underlying immunologic mechanisms of tumorigenesis and tumor evasion has prompted the evaluation of additional receptors involved in the $\mathrm{T}$ cell response. Studies of agonist antibodies targeting the immune-stimulatory receptors OX40, CD27, CD137 and GITR are awaited. Similarly, promising results have been suggested by early-phase clinical trials investigating molecules targeting the inhibitory co-receptors LAG-3 and TIM-3, as well as indeoleamine 2,3-dioxygenase (IDO) inhibition, a tryptophan-metabolizing enzyme involved in immunosuppressive mechanisms. ${ }^{45}$

Another promising approach already approved for clinical use in the USA and Europe is talimogene laherparepvec (T-VEC), an oncolytic attenuated herpes virus designed to selectively replicate and lyse tumor cells and overexpress granulocyte macrophage colony-stimulating factor (GM-CSF), resulting in induction of tumor-specific $\mathrm{T}$ cell response. In a phase 3 trial with patients with stage IIIB-IV melanoma, intratumoral injections of T-VEC produced an improved durable response rate compared to intratumoral GM-CSF alone (16.3\% vs. $2.1 \%$; OR 8.9; $\mathrm{p}<0.001$ ), leading to its approval by regulatory agencies. ${ }^{46}$ Phase $1 \mathrm{~b}$ studies tested combinations of T-VEC with systemic immunotherapies, revealing a safe profile and interesting results. T-VEC in association with ipilimumab produced an ORR of $50 \%$, with $44 \%$ of the patients having durable responses of at least 6 months; the 18-month overall survival was $67 \%{ }^{47}$ The combination of TVEC with 
pembrolizumab achieved an ORR of $57.1 \%$, with $23.8 \%$ having confirmed complete responses. This strategy is now being further evaluated in an ongoing phase 3 clinical trial (MASTERKEY265; NCT02263508). ${ }^{48}$

Another attractive approach involves combining anti-PD1/PDL-1 molecules with BRAF/MEK inhibitors. In a phase 1 dose-escalation study, patients received atezolizumab, an anti-PD-L1 agent, in association with vemurafenib. The trial demonstrated a manageable toxicity profile and promising antitumor activity, with an ORR of $76 \%$ and a median PFS of 10.9 months. ${ }^{49}$ The triplet combination of pembrolizumab, dabrafenib and trametinib has also been shown to be feasible: although $67 \%$ of the patients experienced grade 3-4 adverse events, leading to discontinuation of treatment in $33 \%$ of the cases, this combination resulted in an ORR of $60 \% .^{50}$

\section{Current treatment options for patients With adVANCEd MELANoma IN Brazil - Same DISEASE, DIFFERENT PERSPECTIVES}

Advances in the past 5 years have widened the treatment possibilities for advanced melanoma patients, with an undeniable impact on overall survival (Figure 3 and Table 1). In the USA and Europe, those with BRAF-mutated tumors can be treated, in the first line setting, with the anti$\mathrm{BRAF} / \mathrm{MEK}$ combinations vemurafenib/cobimetinib or dabrafenib/trametinib. Also, as mentioned before, immunotherapy with nivolumab, pembrolizumab, ipilimumab/ nivolumab, ipilimumab or T-VEC is approved and available.

In Brazil, while nivolumab and pembrolizumab have been approved in 2016, the combination of ipilimumab and nivolumab has not been incorporated to date, and ipilimumab has been approved only for patients who have failed a first-line therapy. Similarly, for molecularly-selected patients, additional options include dabrafenib used as single-agent, vemurafenib as single agent or the combination of vemurafenib and cobimetinib. Quite concerning, however, is the fact that none of the mentioned approved therapies is available in the public health system, in which treatment still relies on standard cytotoxic chemotherapies.

If it is true that predictive and prognostic biomarkers can help in a better patient selection in a setting of skyrocketing costs associated with cancer care and often limiting toxicities, the only validated biomarker ready for unrestricted clinical use and that can direct treatment decisions is the assessment of the BRAF status. Factors involved in the antitumor immune response could potentially identify the best candidates for immune-checkpoint blockade, and many are being extensively investi- gated: total tumor mutational load, tumor peptidome, expression of PD-L1, clonality of the TCR, density and quality of immune infiltrates, gene expression profiles associated with an "inflamed" phenotype, genomic determinants of antitumor immunity and even the characterization of commensal bacteria that could potentially modulate cell-mediated responses. A better characterization of the mechanisms involved in primary and secondary resistance to either immunotherapy or targeted therapy is also mandatory for a rational development of future treatment strategies and compounds.

\section{Conclusion}

Therapies for patients with advanced melanoma have rapidly evolved over the past few years, improving quality of life and life expectancy. Checkpoint inhibition with antibodies directed against PD-1, nivolumab and pembrolizumab, alone or in combination with the anti-CTLA4 agent ipilimumab, has become the preferred approach for patients with advanced melanoma not harboring BRAF mutations. Molecularly-targeted therapies directed against the MAPK pathway also provide additional options for those with a BRAF V600 mutation, and the association of MEK inhibitors to BRAF inhibitors produced increased response rates, progression free survival and overall survival, and rational ways to combine and sequence this armamentarium will most likely allow for an even greater impact on survival for these patients. Although directed therapies have not been approved for non-BRAF molecular aberrations, including KIT and NRAS mutations, high expectations for the coming years are justifiable by both ongoing pre-clinical and clinical development. Translational research and future clinical trials are warranted to address the large body of questions that remain to be answered, and strategies to bring this reality to in a cost-effective manner to countries with significant cost contingency issues are mandatory.

\section{Conflict of interest}

The authors have the following conflicts of interest (COI) to disclose:

- A.H. - No COI to disclose

- A.S. - No COI to disclose

- C.A.A - Honoraria (BMS, MSD, Roche); travel expenses (MSD, Novartis, Roche); advisory role (BMS, MSD)

- M.S. - Honoraria (MSD); advisory role (MSD, Roche); travel expenses (BMS, MSD, Roche)

- V.P.C. - Honoraria (BMS, MSD, Novartis)

- B.G. - Honoraria (BMS, Janssen, MSD); advisory role (BMS, Janssen, MSD) 
- M.A.P. - Honoraria (BMS, Merck); advisory role (BMS, Amgen); research funding (BMS)

- G.S.F. - Honoraria (Novartis, Mundipharma, Roche); advisory role (Mundipharma, Roche, Servier); travel expenses (Bayer, Mundipharma, Novartis, Roche, Servier); research involvement (BMS, MSD)

- R.R.M. - Honoraria (AstraZeneca, BMS, MSD, Roche, Novartis); advisory role (Roche, MSD); travel expenses (AstraZeneca, BMS, MSD, Roche, TEVA, Novartis); research involvement (Lilly, Roche)

\section{Resumo}

Tratamento de melanoma avançado - Um panorama em transformação

Após décadas de ostracismo, os recentes avanços no tratamento do melanoma trouxeram uma nova realidade para pacientes, médicos e pesquisadores. Enquanto anticorpos monoclonais voltados a moléculas envolvidas na modulação da interação entre células do melanoma e do sistema imune consolidaram o uso da "imunoterapia", um melhor conhecimento acerca das aberrações genômicas envolvidas na carcinogênese do melanoma viabilizaram o desenvolvimento de inibidores da via mitogen-activated protein kinase pathway (MAPK), o que também resultou em ganhos significativos em taxas de resposta e sobrevida. Consequentemente, novas modalidades de tratamento foram aprovadas para uso clínico nos Estados Unidos e na Europa, incluindo os bloqueadores de correceptores imunes ipilimumabe, nivolumabe e pembrolizumabe, o herpesvírus oncolítico talimogene laherparepvec (T-VEC), e os agentes-alvo vemurafenibe, dabrafenibe, cobimetinibe e trametinibe. Nesse artigo, revisamos os resultados que trouxeram novas alternativas para a prática clínica e discutimos a incorporação desses avanços ao cuidado de pacientes no Brasil.

Palavras-chave: melanoma, anti-PD1, anti-CTLA-4, BRAF, MEK.

\section{References}

1. American Cancer Society. Cancer Facts and Figures 2016 [cited 2016 Oct 14]. Available from: http://www.cancer.org/acs/groups/content/@research/ documents/document/acspc-047079.pdf.

2. Erdei E, Torres SM. A new understanding in the epidemiology of melanoma. Expert Rev Anticancer Ther. 2010; 10(11):1811-23.

3. Schmerling RA, Loria D, Cinat G, Ramos WE, Cardona AF, Sánchez JL, et al. Cutaneous melanoma in Latin America: the need for more data. Rev Panam Salud Publica. 2011; 30(5):431-8.

4. INCA. Instituto Nacional do Câncer. Estimativa 2016. Incidência d0 câncer no Brasil. [cited 2016 Oct 14]. Available from: http://www.inca.gov.br/ estimativa/2016/estimativa-2016-v11.pdf.
5. Bhatia S, Tykodi SS, Thompson JA. Treatment of metastatic melanoma: an overview. Oncology. 2009; 23(6):488-96

6. Atkins MB, Kunkel L, Sznol M, Rosenberg SA. High-dose recombinant interleukin-2 therapy in patients with metastatic melanoma: long-term survival update. Cancer J Sci Am. 2000; 6(Suppl 1):S11-4.

7. Yajima I, Kumasaka MY, Thang ND, Goto Y, Takeda K, Yamanoshita O, et al. RAS/RAF/MEK/ERK and PI3K/PTEN/AKT signaling in malignant melanoma progression and therapy. Dermatol Res Pract. 2012; 2012:354191.

8. Kim KB, Kefford R, Pavlick AC, Infante JR, Ribas A, Sosman JA et al. Phase II study of the MEK1/MEK2 inhibitor Trametinib in patients with metastatic BRAF-mutant cutaneous melanoma previously treated with or without a BRAF inhibitor. J Clin Oncol. 2013; 31(4):482-9.

9. The Cancer Genome Atlas Network. Genomic classification of cutaneous melanoma. Cell. 2015; 161(7):1681-96.

10. Hodi FS, Kluger H, Sznol M, Carvajal R, Lawrence D, Atkins M, et al. Durable, long-term survival in previously treated patients with advanced melanoma (MEL) who received nivolumab (NIVO) monotherapy in a phase I trial. Cancer Res. 2016; 76(14 Suppl):abst CT001.

11. Long GV, Menzies AM, Nagrial AM, Haydu LE, Hamilton AL, Mann GJ, et al. Prognostic and clinicopathologic associations of oncogenic BRAF in metastatic melanoma. J Clin Oncol. 2011; 29(10):1239-46.

12. Flaherty KT, Lee SJ, Schuchter LM, Flaherty LE, Wright JJ, Leming PD, et al Final results of E2603: a double-blind, randomized phase III trial comparing carboplatin (C)/paclitaxel (P) with or without sorafenib (S) in metastatic melanoma. J Clin Oncol. 2010; 28(Suppl):613s.

13. McArthur GA, Chapman PB, Robert C, Larkin J, Haanen JB, Dummer R, et al. Safety and efficacy of vemurafenib in BRAF (V600E) and BRAF (V600F) mutation-positive melanoma (BRIM-3): extended follow-up of a phase 3 randomized, open-label study. Lancet Oncol. 2014; 15(3):323-32.

14. Hauschild A, Grob JJ, Demidov LV, Jouary T, Gutzmer R, Millward M, et al. Dabrafenib in BRAF-mutated metastatic melanoma: a multicentre, openlabel, phase 3 randomised controlled trial. Lancet. 2012; 380(9839):358-65.

15. Hauschild A, Grob JJ, Demidov LV, Jouary T, Gutzmer R, Millward M, et al. An update on BREAK-3, a phase III, randomized trial: Dabrafenib versus dacarbazine in patients with BRAF V600E-positive mutation metastatic melanoma. J Clin Oncol. 2013; 31(Suppl):abstr9013. [2013 ASCO Annual Meeting].

16. Rizos H, Menzies AM, Pupo GM, Carlino MS, Fung C, Hyman J, et al. BRAF inhibitor resistance mechanisms in metastatic melanoma: spectrum and clinical impact. Clin Cancer Res. 2014; 20(7):1965-77.

17. Flaherty KT, Robert C, Hersey P, Nathan P, Garbe C, Milhem M, et al.; METRIC Study Group. Improved survival with MEK inhibition in BRAF mutated melanoma. N Eng J Med. 2012; 367(2):107-14.

18. Robert C, Karaszewska B, Schachter J, Rutkowski P, Mackiewicz A Stroiakovski D, et al. Improved overall survival in melanoma with combined dabrafenib and trametinib. N Engl J Med. 2015; 372(1):30-9.

19. Ascierto PA, McArthur GA, Dréno B, Atkinson V, Liszkay G, Di Giacomo $\mathrm{AM}$, et al. Cobimetinib combined with vemurafenib in advanced BRAFV600mutant melanoma (coBRIM): updated efficacy results from a randomised, double-blind, phase 3 trial. Lancet Oncol. 2016; 17(9):1248-60.

20. Long GV,Stroyakovsky DL, Gogas H, Levchenko E, de Braud F, Larkin J, et al. COMBI-d: A randomized, double-blinded, Phase III study comparing the combination of dabrafenib and trametinib to dabrafenib and trametinib placebo as first-line therapy in patients (pts) with unressectable or metastatic BRAFV600E/K mutation-positive cutaneous melanoma. J Clin Oncol. 2014; 32 (Suppl):abstr9011.[2014 ASCO Annual Meeting]

21. Grob JJ, Amonkar MM, Karaszemska B, Schacther J, Dummer R, Mackiewicz A, et al. Comparasion of dabrafenib and trametinib combination therapy with vemurafenib monotherapy on health-realted quality of life in patients with unresectable or metastatic cutaneous BRAFal600-mutation-positive melanoma (COMBI-v): results of a phase 3 , open-label, randomized trial. Lancet Oncol. 2015; 16(13):1389-98.

22. Flaherty K, Davies MA, Grob JJ, Long GV, Nathan PD, Ribas A, et al. Genomic analysis and 3-y efficacy and safety update of COMBI-d: a phase 3 study of dabrafenib (D) + trametinib (T) vs D monotherapy in patients (pts) with unresectable or metastatic BRAF V600E/K mutant cutaneous melanoma. J Clin Oncol. 2016; 34(Suppl):abstr9502. [2016 ASCO Annual Meeting]

23. Robert C, Karazewska B, Schachter J, Rutkowski P, Mackiewicz A, Stroyakovsiy $\mathrm{D}$, et al. Three-year estimate of overall survival in COMBI-v, a randomized phase 3 study evaluating first-line dabrafenib (D) + trametinib (T) in patients (pts) with unresectable or metastatic BRAF V600E/K-mutant cutaneous melanoma. Ann Oncol. 2016; 27(Suppl 6):LBA40. 
24. Dummer R, Schadendorf D, Ascierto PA, Fernández AMA, Dutriaux C, Maio $\mathrm{M}$, et al. Results of NEMO: a phase III trial of binimetinib (BINI) vs dacarbazine (DTIC) in NRAS-mutant cutaneous melanoma. J Clin Oncol. 2016; 34(Suppl):abstr9500). [2016 ASCO Annual Meeting]

25. Hodi FS, Corless CL, Giobbie-Hurder A, Fletcher JA, Zhu M, Marino-Enriquez A, et al. Imatinib for melanomas harboring mutationally activated or amplified KIT arising on mucosal, acral, and chronically sun-damaged skin. J Clin Oncol. 2013; 31(26):3182-90.

26. Carvajal RD, Antonescu CR, Wolchock JD, Chapman PB, Roman RA, Teitcher J, et al. KIT as a therapeutic target in metastatic melanoma. JAMA. 2011; 305(22):2327-34

27. Kim KB, Eton O, Davis DW, Frazier ML, McConkey DJ, Diwan AH, et al. Phase II trial of imatinib mesylate in patients with metastatic melanoma. Br J Cancer. 2008; 99(5):734-40.

28. Guo J, Si L, Kong Y, Flaherty KT, Xu X, Zhu Y, et al. Phase II, open-label, singlearm trial of imatinib mesylate in patients with metastatic melanoma harboring c-KIT mutation or amplification. J Clin Oncol. 2011;29(21):2904-9.

29. Hennecke J, Wiley DC. T cell receptor-MHC interactions up close. Cell. 2001; 104(1):1-4.

30. Chen L, Flies DB. Molecular mechanisms of T cell co-stimulation and coinhibition. Nat Rev Immunol. 2013; 13(4):227-42.

31. Hodi FS, O'Day SJ, McDermott DF, Weber RW, Sosman JA, Haanen JB, et al. Improved survival with ipilimumab in patients with metastatic melanoma. N Engl J Med. 2010; 3638):711-23

32. Schadendorf D, Hodi FS, Robert C, Weber IS, Margolin K, Hamid O, et al. Pooled analysis of long-term survival data from Phase II and Phase III trials of ipilimumab in unresectable or metastatic melanoma. J Clin Oncol. 2015; 33(17):1889-94.

33. Ascierto PA, Del Vecchio M, Robert C, Mackiewcz A, Chiarion-Sileni V, Arance Fernandez AM, et al. Overall survival (OS) and safety results from a phase 3 trial of ipilimumab (IPI) at $3 \mathrm{mg} / \mathrm{kg}$ vs $10 \mathrm{mg} / \mathrm{kg}$ in patients with metastatic melanoma (MEL). In: ESMO, 2016, Copenhagen. Annals of Oncology, Copenhagen, Denmark, Oxford University Press, 2016. Abstr. vi379.

34. Ribas A, Hamid O, Daud A, Hodi FS, Wolchok J D, Kefford R, et al. Association of Pembrolizumab with tumor response and survival among patients with advanced melanoma. JAMA. 2016; 315(15):1600-9.

35. Topalian SL, Sznol M, McDermott DF, Kluger HM, Carvajal RD, Sharfman $\mathrm{WH}$, et al. Survival, durable tumor remission, and long-term safety in patients with advanced melanoma receiving nivolumab. J Clin Oncol. 2014; 32(10):1020-30

36. Ribas A, Puzanov I, Dummer R, Scadendorf D, Hamid O, Robert C, et al. Pembrolizumab versus investigator-choice chemotherapy for ipilimumabrefractory melanoma (KEYNOTE-002): a randomised, controlled, phase 2 trial. Lancet Oncol. 2015; 16(8):908-18.

37. Weber JS, D’Angelo SP, Minor D, Hodi FS, Gutzmer R, Neyns B, et al. Nivolumab versus chemotherapy in patients with advanced melanoma who progressed after anti-CTLA-4-treatment (CheckMate 037): a randomised, controlled, open-label, phase 3 trial. Lancet Oncol. 2015; 16(4):375-84.
38. Daud A, Blank CU, Robert C, Puzanov I, Richtig E, Margolin KA, et al. KEYNOTE-006 study of pembrolizumab (pembro) versus ipilimumab (ipi) for advanced melanoma: efficacy by PD-L1 expression and line of therapy. J Clin Oncol. 2016; 34(Suppl):abstr9513. [2016 ASCO Annual Meeting]

39. Schacter J, Ribas A, Ling GV, Arance A, Grob JJ, Mortier L, et al. Pembrolizumab versus ipilimumab for advanced melanoma: Final overall survival analysis of KEYNOTE-006. J Clin Oncol. 2016; 34(Suppl):abstr9504. [2016 ASCO Annual Meeting]

40. Postow MA, Chesney J, Pavlick AC, Robert C, Grossmann K, McDermott D, et al. Nivolumab and ipilimumab versus ipilimumab in untreated melanoma. N Engl J Med. 2015; 372(21):2006-17.

41. Larkin J, Chiarion-Sileni V, Gonzalez R, Grob JJ, Cowey CL, Lao CD, et al. Combined Nivolumab and Ipilimumab or monotherapy in untreated melanoma. N Engl J Med. 2015; 373(1):23-34.

42. Wolchok JD, Chiarion-Sileni V, Gonzalez R, Rutkowski P, Grob JJ, Cowey CL, et al. Updated results from a phase III trial of nivolumab (NIVO) combined with ipilimumab (IPI) in treatment-naive patients (pts) with advanced melanoma (MEL) (CheckMate 067). J Clin Oncol. 2016; 34(Suppl):abstr9505. [2016 ASCO Annual Meeting]

43. Long GV, Atkinson V, Cebon JS, Jameson MB, Fitzharris BM, McNeil CM, et al. Pembrolizumab (pembro) plus ipilimumab (ipi) for advanced melanoma: Results of the KEYNOTE-029 expansion cohort. J Clin Oncol. 34, 2016; 34Suppl):abstr9506. [2016 ASCO Annual Meeting]

44. Dummer R, Hauschild A, Lindenblatt N, Pentheroudakis G, Keilholz U. Cutaneous melanoma: ESMO Clinical Practice Guidelines for diagnosis, treatment and follow-up. Ann Oncol. 2015; 26(Suppl 5):v126-32.

45. Mellman I, Coukos G, Dranoff G. Cancer immunotherapy comes of age Nature. 2011; 480(7378):480-9.

46. Andtbacka RH, Kaufman HL, Collichio F, Amatruda T, Senzer N, Chesney $\mathrm{J}$, et al. Talimogene Laherparepvec improves durable response rate in patients with advanced melanoma. J Clin Oncol. 2015; 33(25):2780-8.

47. Puzanov I, Milhem MM, Minor D, Hamid O, Li A, Chen L, et al. Talimogene Laherparepvec in combination with ipilimumab in previously untreated, unresectable stage IIIB-IV melanoma. J Clin Oncol. 2016; 34(22):2619-26.

48. Long GV, Dummer R, Ribas A, Puzanov I, VanderWalde A, Hans R, Andtbacka I, et al. Efficacy analysis of MASTERKEY-265 phase $1 \mathrm{~b}$ study of talimogene laherparepvec (T-VEC) and pembrolizumab (pembro) for unresectable stage IIIB-IV melanoma. J Clin Oncol. 2016; 34(Suppl):abstr9568. [2016 ASCO Annual Meeting].

49. Hamid O, Patel M, Hodi S, Amaria R, Boasberg P, Sullivan R, et al. Preliminary clinical safety, tolerability and activity of atezolizumab (anti-PD-L1) combined with Zelboraf in BRAFv600 metastatic melanoma. Presented at the 12 Congress of the Society for Melanoma Research 2015; November 18-21, 2015; San Francisco, CA. Abstract.

50. Ribas A, Hodi FS, Lawrence DP, Atkinson V, Starodub A, Carlino MS, et al Pembrolizumab (pembro) in combination with dabrafenib (D) and trametinib (T) for BRAF-mutant advanced melanoma: Phase 1 KEYNOTE-022 study. J Clin Oncol. 2016; 34(Suppl):abstr 3014. [2016 ASCO Annual Meeting]. 